\author{
TREX13 data analysis/modeling \\ Dajun (DJ) Tang \\ Applied Physics Laboratory, University of Washington \\ 1013 NE $40^{\text {th }}$ Street \\ Seattle, WA 98105 \\ phone: (206) 543-1290 fax: (206) 543-6785 email: djtang@apl.washington.edu
}

Award Number: N00014-14-1-0239

CO-PI's: Jie Yang (jieyang@apl.washington.edu)

Frank Henyey (frank@apl.washington.edu)

\title{
LONG-TERM GOALS
}

To understand mid-frequency $(1-10 \mathrm{kHz})$ acoustics in shallow water through measurements and modeling, including propagation, reflection, and forward- and backscatter, as well as reverberation. The top-level goals of this effort are to understand the important environmental processes that impact mid-frequency sonar performance in shallow waters.

\section{OBJECTIVES}

TREX13 (Target and REverberation eXperiment) was conducted during April-June, 2013 off the coast of Panama City, Florida where the water depth is approximately $20 \mathrm{~m}$. The frequency range covered is 1-10 kHz, emphasizing 3-4 kHz. The Navy relevance is reflected in the fact that detection using midfrequency sonar is in most cases reverberation limited. This project addresses a clear need for a 6.1 level program, i.e., using well-controlled geometries and high resolution environmental measurements (1) to test models predicting reverberation and (2) to quantify the most important environmental measurements to make in order to maintain accuracy in those predictions. With extensive TREX13 data in hand, the objective now shifts to realizing the long-term goals using data analysis and modeling.

\section{APPROACH}

While the TREX13 data sets are extensive and cover almost all aspects of shallow water acoustics, a central theme based on a SONAR equation as given in Fig. 1 summaries the approach to data analysis and modeling philosophy. This figure is from a page of the TRX13 web site under development at APL-UW. The SONAR equation appropriate for the project is

$$
\mathrm{RL}=\mathrm{SL}-2 \mathrm{x} \mathrm{TL}+\mathrm{ISS}
$$

where RL is reverberation level, SL the source level, 2xTL the two-way transmission loss, and ISS the scattering strength integrated over the scattering patch for given sonar beam. Two unique features of TREX13 are that: 1) all components of the SONAR equation are individually measured in the same frequency band over the same environment, 2) an extensive environmental measurements at the appropriate temporal and spatial resolutions were made such that basic research questions concerning 


\section{Report Documentation Page}

Form Approved

OMB No. 0704-0188

Public reporting burden for the collection of information is estimated to average 1 hour per response, including the time for reviewing instructions, searching existing data sources, gathering and maintaining the data needed, and completing and reviewing the collection of information. Send comments regarding this burden estimate or any other aspect of this collection of information,

including suggestions for reducing this burden, to Washington Headquarters Services, Directorate for Information Operations and Reports, 1215 Jefferson Davis Highway, Suite 1204, Arlington

VA 22202-4302. Respondents should be aware that notwithstanding any other provision of law, no person shall be subject to a penalty for failing to comply with a collection of information if it

does not display a currently valid OMB control number.

1. REPORT DATE

30 SEP 2014

4. TITLE AND SUBTITLE

TREX13 Data Analysis/modeling

6. $\operatorname{AUTHOR}(\mathrm{S})$

7. PERFORMING ORGANIZATION NAME(S) AND ADDRESS(ES)

University of Washington,Applied Physics Laboratory,1013 NE 40th Street,Seattle,WA,98105

9. SPONSORING/MONITORING AGENCY NAME(S) AND ADDRESS(ES)
3. DATES COVERED

00-00-2014 to 00-00-2014

5a. CONTRACT NUMBER

5b. GRANT NUMBER

5c. PROGRAM ELEMENT NUMBER

5d. PROJECT NUMBER

5e. TASK NUMBER

5f. WORK UNIT NUMBER

8. PERFORMING ORGANIZATION

REPORT NUMBER

10. SPONSOR/MONITOR'S ACRONYM(S)

11. SPONSOR/MONITOR'S REPORT

NUMBER(S)

12. DISTRIBUTION/AVAILABILITY STATEMENT

Approved for public release; distribution unlimited

13. SUPPLEMENTARY NOTES

14. ABSTRACT

15. SUBJECT TERMS

16. SECURITY CLASSIFICATION OF: a. REPORT

unclassified b. ABSTRACT

unclassified c. THIS PAGE

unclassified
17. LIMITATION OF ABSTRACT

Same as

Report (SAR)
18. NUMBER 19a. NAME OF

OF PAGES

9 
predictability and uncertainty of shallow water reverberation can be quantitatively addressed. The approach to data analysis can be summarized into the following steps:

1. Based only on acoustic measurements, assess to what degree the measured reverberation, transmission, and scattering quantities satisfy the SONAR equation? This first step establishes a complete data set that enables detailed follow-up analysis. It also bounds the predictability and uncertainty of reverberation.

2. Incorporating environmental data, assess the predictability and uncertainty of the individual terms in the SONAR equation. Identify key environmental parameters that contribute to the variability.

3. Review available reverberation predictive models, and if necessary, develop new models to incorporate environmental knowledge in order to improve model accuracy and/or speed.

4. With both acoustics and environments measured, divide model environmental parameters into categories, e. g., those that can be inferred from acoustic data and those where databases are necessary.

5. Given prediction requirements and uncertainty tolerance, provide a set of key environmental parameters necessary as input to models.

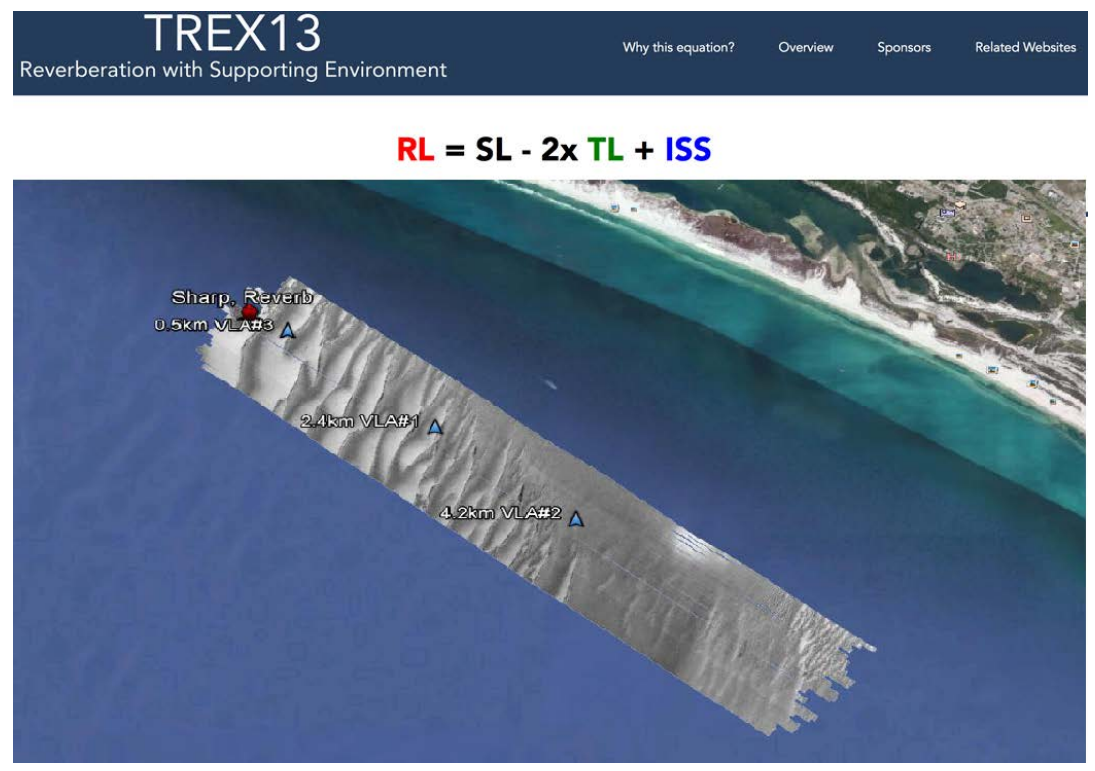

Figure 1. Schematic of TREX13 based on TREX13 website under development.

The $400 \mathrm{kHz}$ backscatter strength from the multibeam survey (de Moustier) is shown as the gray swath along the Main Reverberation Track where focused environmental characterization was conducted. Main acoustic assets are shown here: The reverberation sources and receiving arrays are deployed from the $R / V$ Sharp and are labeled "Sharp, Reverb". The three vertical line arrays fielded by Scripps Institution of Oceanography are shown as triangles with labels given distances to the reverberation source. 


\section{WORK COMPLETED}

The discussion on work completed will be divided into each term of the SONAR equation, i.e., the reverberation level (RL), transmission loss (TL), and the integrated scattering strength (ISS).

Reverberation Level (RL)

Jie Yang took the lead in analysis of the reverberation data. Over the duration of TREX13, RL data were collected daily, resulting in a large number of data sets which spans over several weeks and under varying environmental conditions. To address different physics issues, we have divided the data sets into four categories: (1) Reverberation under calm sea surface conditions with low ambient noise and low biological activity. Data in this category is especially suitable for studying reverberation dominated by bottom scattering. (2) Reverberation under different sea surface conditions, including calm condition and rough sea surface conditions when surface waves move in different directions relative to the sonar beam. Data in this category is meant for detailed study of wind impact on reverberation (See report by Throsos). (3) Reverberation under the influence of high bio-clutter and ambient noise. Fish are frequently present in the TREX13 scene and often a major reverberation source. Fish effect is especially notable after dusk and before sunrise. The TREX13 site is close to a channel so shipping noise is often a concern. In addition, we have noticed that noise generated by fish increase ambient noise level by as much as $15 \mathrm{~dB}$ over a short time ( $<20$ minutes). Reverberation data "contaminated" by fish and noise are carefully grouped. Data in this category will be suitable for SONAR performance analysis under these environmental conditions. (4) Target detection under varying environmental condition. An air-filled hose was fielded by DRDC, Canada, to serve as a known target. In addition, DRDC also deployed an echo repeater from a moving ship to work in conjunction with the reverberation measurements, mimicking a moving target. These target data are reserved for evaluating SONAR performance after reverberation data analysis for the first three categories are finished. As examples, Fig. 2 shows overnight data for different nights under varying environmental conditions, ranging from calm condition (upper left), mild biological activity (upper right), heavy biological activity (lower left), and heavy shipping (lower right).
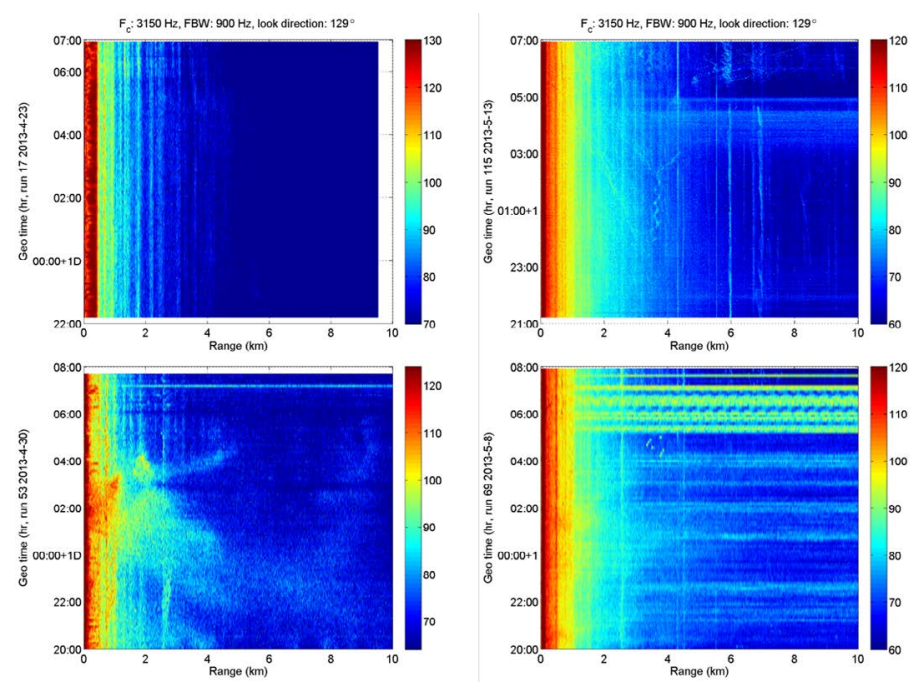

Figure 2. Reverberation level measured along the main reverberation track over four different nights: calm condition (upper left), mild biological activity (upper right), heavy biological activity (lower left), and heavy shipping noise (lower right). 


\section{Transmission Loss (TL)}

During TREX13, three separate kinds of TL data were collected for the purpose of modeling two-way propagation in conjunction with reverberation, each has its unique advantages and suitable for different aspects of TL modeling. The first kind is transmission from the reverberation source at the R/V Sharp site to the three Scripps vertical line arrays, as depicted in Fig. 1. The special feature of these data is that these are the same pings from which reverberation is also measured, although TL for only three ranges, $0.5 \mathrm{~km}, 2.4 \mathrm{~km}$, and $4.2 \mathrm{~km}$, are available. The second kind TL measurement is source-tow by a moving ship, conducted by Scripps. The source-tow track is shown in Fig. 3, along with the three vertical line arrays as receivers. These source tow data on the VLAs cover propagation paths which overlap with the reverberation path and cover a substantial portion of the water column, hence providing ideal data for modeling TL. The third kind TL measurement is by using a moving ship with an echo repeater. This part is collaboration with DRDC, Canada. Fig.4 shows an example of the echo repeater run: Each reverberation transmission from the Sharp is received by the moving ship; a source on the moving ship re-transmits the received signal back to the source, mimicking a target echo. TL data of this kind are especially suited for analysis of target detection under reverberation.

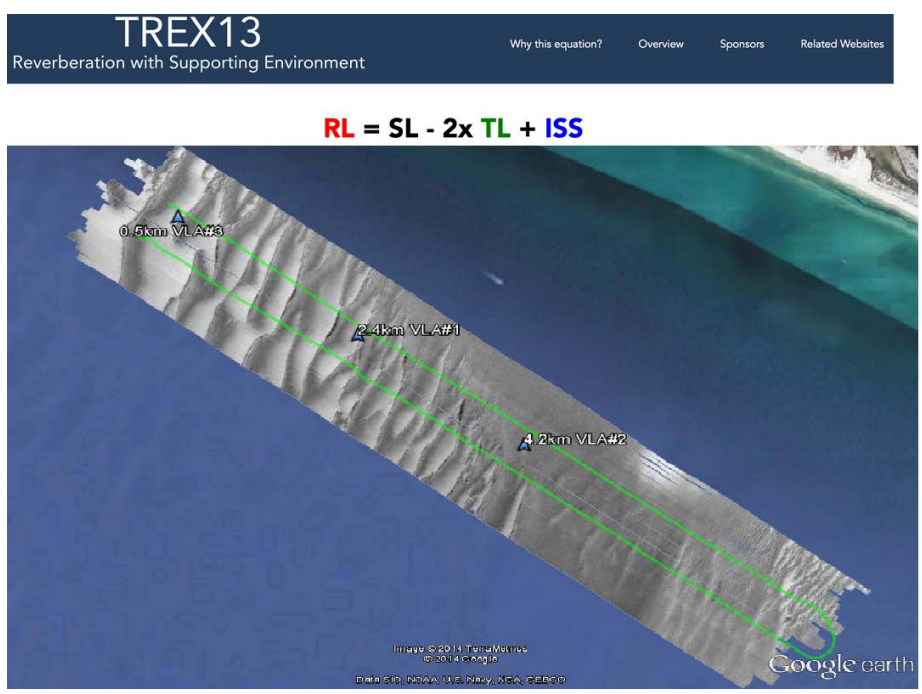

Figure 3. Background information is the same as for Figure 1. The green curve shows one of the Scripps source-tow track for TL measurement on the VLAs'. 


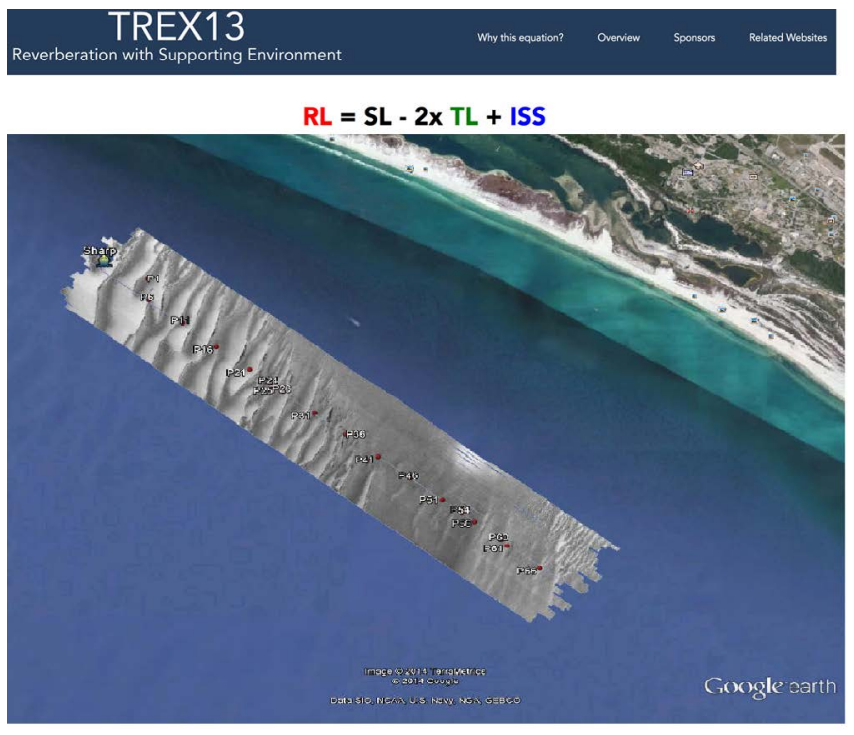

Figure 4. Background information is the same as for Figure 1. The red dots showing the DRDC echo repeater ping locations for every $5^{\text {th }}$ ping, echoing transmitions from the reverberation source at the $R / V$ Sharp site.

ISS

A portable system called Bottom Backscatter SONAR (BSS) is used to measure direct path bottom backscatter and normal incident reflection at different locations along the reverberation track. It consists of a single source and single receiver, and the measurements are made on a hired 30 -ft-long dive boat. The measurement strategy is to target the transition regions observed in the multibeam data, which are the dark black strips in Fig. 5. To that end, the dive boat is steamed to a position near a transition region where the prevailing current would make the boat drift across the transition region. When a pre-selected starting location is arrived at, the engine of the boat is turned off and the boat is free drifting. The course of the boat is tracked by a GPS receiver as a function of time, and acoustic transmission time is also recorded, hence the locations of each transmission can be derived. The uncertainty of the locations is on the order of $10 \mathrm{~m}$. While in drift mode, the sensor bracket of the BSS is lowered by a line from the stern of the boat to a depth roughly 2-5 $\mathrm{m}$ above the bottom, taking data. Fig. 5 shows all the drifting tracks where the BSS took data. It covers several of the transition regions along the main reverberation track, and also includes a track toward the southwest at a range of $\sim 5 \mathrm{~km}$ where the sediment is appears uniform in the multibeam data. The drifting tracks are about 100-250 m in length and each contains 500-1000 repetitions of the same waveform. The prevailing current of the testing day is such that the drift tracks all tend to be perpendicular to shore and almost parallel to the transition regions, with several exceptions at the NW end. There the tracks are closer to 45 degrees from parallel to the transition regions. 


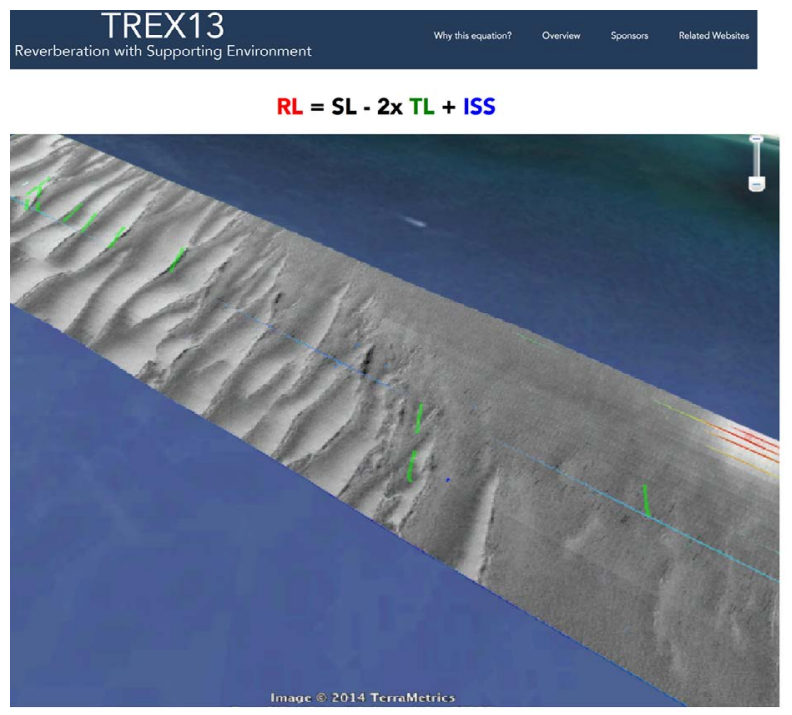

Figure 5. The gray background is the scattering strength from the $400 \mathrm{kHz}$ multibeam data and the
green short tracks show the locations of measurements

\section{RESULTS}

The main results on RL, TL, and ISS are summarized below. Fig. 6 gives the RL under calm conditions for a $100 \mathrm{~Hz}$ band signal centered around $3.5 \mathrm{kHz}$. The key result is that while the general trend of RL vs range is familiar to those who have made reverberation observations in shallow waters, there exist fluctuations on the order of $10 \mathrm{~dB}$ in the RL curve for range shorter than $4 \mathrm{~km}$. These fluctuations are not random; rather they coincide with the "transition regions" found in the $400 \mathrm{kHz}$ multibeam images. In addition, these fluctuations are target-like, and statistically behave as clutter. Detailed analysis and modeling of the RL behavior will be the next step. Date and modeling results on reverberation under different wind conditions are given in report by Thorsos.

Analysis on TL data indicates that the overall trend in TL is consistent with model predictions using minimum environmental data assuming a typical sandy bottom. Interestingly, however, there exist definite spatial fluctuations on TL over space. An example of TL analysis is given in Fig. 7. An interesting immediate next step is to investigate if the TL fluctuations are also correlated to the "transition regions" as does the RL fluctuations.

Results on ISS from the BSS measurement reveal a close one-to-one correlation of bottom reflectivity to the "transition regions" from the multibeam data. As an example, a cluster of four BSS data tracks are concentrated in the "Transition 1" area, as shown in Fig. 8. All tracks start from the bottom (south) end. Track $a$ is on the southeast of the transition, track $b$ straddle the transition, track $c$ missed almost all the transition except at the very beginning, and track $d$ misses the transition all together. Fig. 9 shows the corresponding reflectivity. The following observation can be made: The reflectivity on either side of the transition has a mean value of around $-10 \mathrm{~dB}$ with minimal variations. The magnitude of reflectivity is consistent with that of sandy sediments. For this see the first half of $a$, second half of $b$, most of $c$ beyond 50m range and all of $d$. Near and over the transition, the reflectivity is variable and generally lower. The fact that both the reverberation level fluctuations and the 
reflectivity variation correlate uniquely to bottom features points toward a unifying underlying physics that controls the variability of the various terms in the SONAR equation.

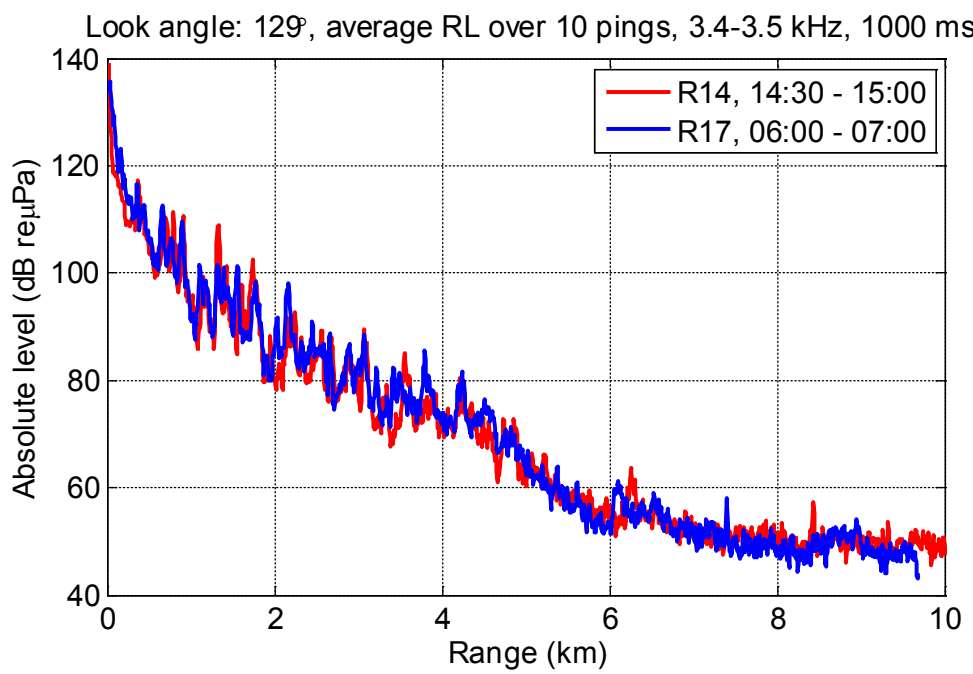

Figure 6. Reverberation Level measured in two different days, both under calm sea conditions. The center frequency is $3450 \mathrm{~Hz}$ and the bandwidth is $100 \mathrm{~Hz}$.

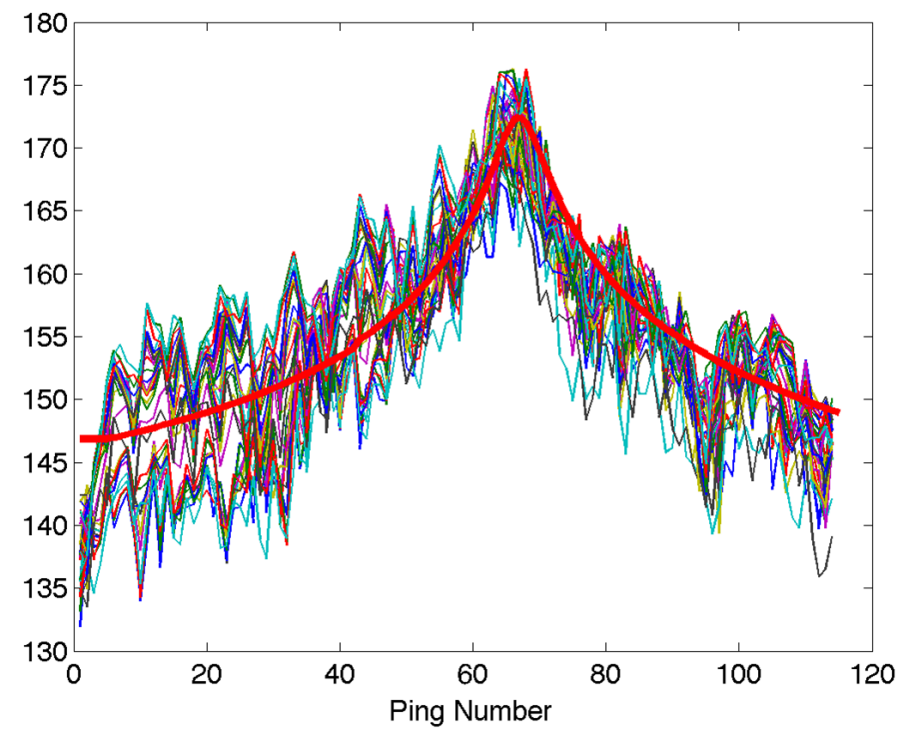

Figure 7. Source-tow TL measurements on Scripps VLA 2 from the lower track shown in Fig. 3. The rapidly varying curves are from the 32 channels of the array. The thick red curve is a model result using normal modes. The vertical axis is received level. The horizontal axis is given as ping numbers, where the peak corresponds to the closest point of approach at approximately 200 m, and ping number 1 is at an approximate range of $3950 \mathrm{~m}$. 


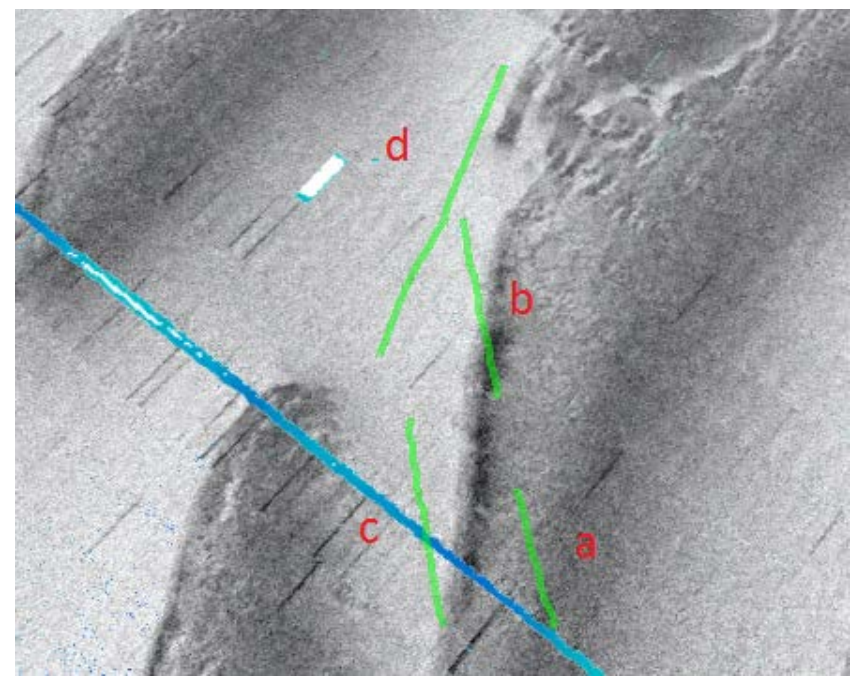

Figure 8. A cluster of 4 data tracks near "transition 1", approximately $860 \mathrm{~m}$ from the moored Sharp where reverberation is measured. The background is the multibeam backscatter, and the very dark stripe is referred to as the transition.
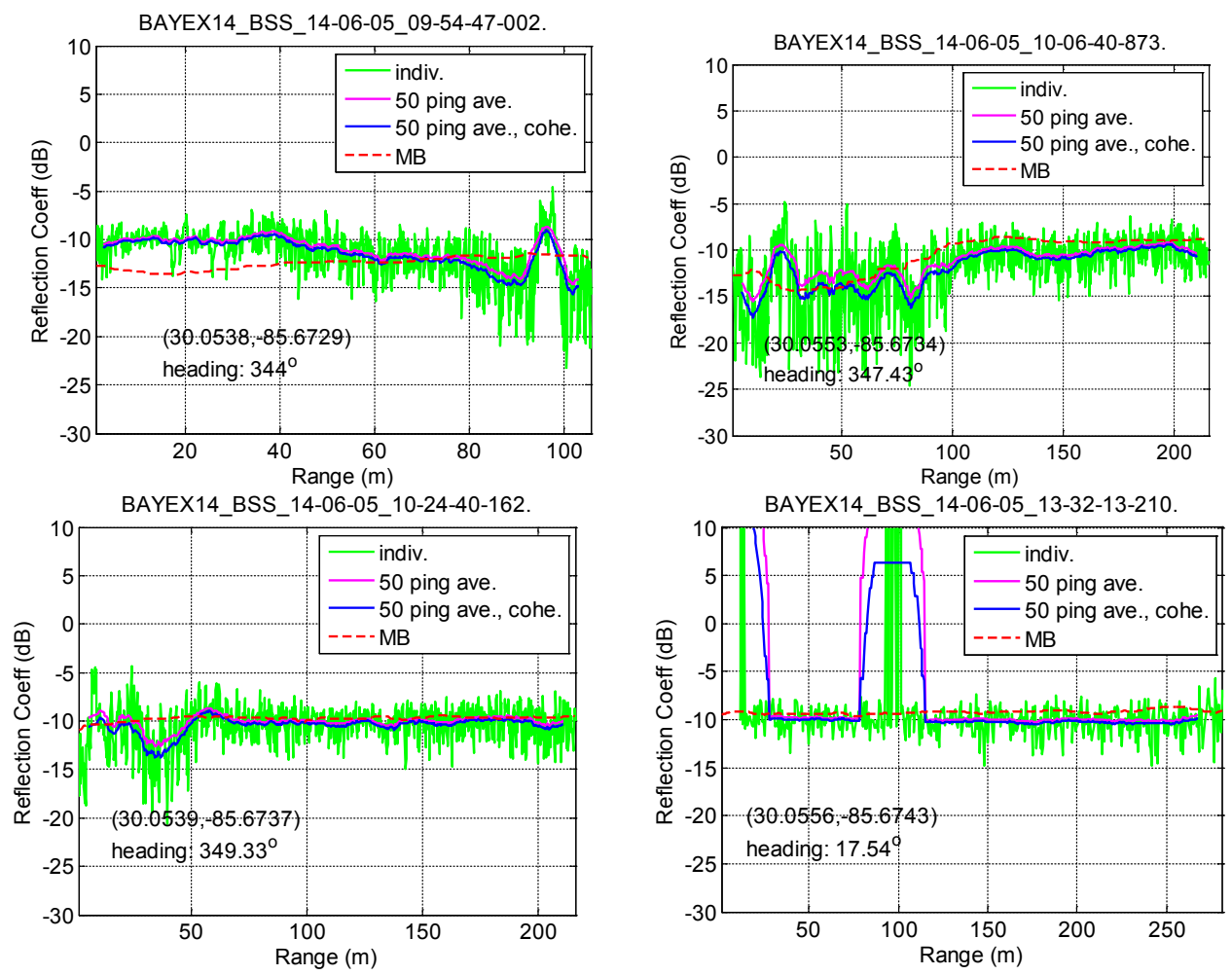

Figure 9. Reflectivity from the four tracks near transition 1, clockwise from top left: a, b, $c$, and d. The very large values such as those near the $100 \mathrm{~m}$ range in the lower right are processing artifacts and should be ignored. 


\section{IMPACT/APPLICATIONS}

Naval active sonar detection is often reverberation limited. Understanding the main mechanisms that cause the diffuse reverberation will lead to better sonar performance.Theoretical and numerical progress inspired by the field work will find applications toward detection in shallow water areas including operational recommendations of the most important environmental measurements to make in order to maintain accuracy in predictions of reverberation.

\section{RELATED PROJECTS}

NAVOCEANO data bases

ONR Signal processing code, active

ONR OA geoacoustic inversion field program

"Reverberation, sediment acoustics, and targets-in-the-environment," ONR Grant \#: N00014-11-10428, PI: K. L. Williams.

"Full Scale Measurement and Modeling of the Acoustic Response of Proud and Buried Munitions at Frequencies from 1-30 kHz," SERDP Contract \#: W912HQ-09-C-0027, PI: S. G. Kargl

\section{PUBLICATIONS}

1. Henyey, F. S., and D. Tang, "Reverberation clutter induced by nonlinear internal waves in shallow water," J. Acoust. Soc. Am. 131, EL302-308 (2013).

2. Tang, D., and D. R. Jackson, “Application of Small-Roughness Perturbation Theory to RangeDependent Waveguides,” J. Acoust. Soc. Am., 134 (4), 289- 293 (2013).

3. Jie Yang, Darrell R. Jackson, and Dajun Tang, "Mid-frequency geoacoustic inversion using bottom loss data from the Shallow Water 2006 Experiment", J. Acoust. Soc. Am. 131 (2), 1711 1721 (2012).

4. Tang, D., and B. T. Hefner, "Modeling backscatter from a series of sediment rough interfaces by a normal incident chirp sonar,"J. Acoust. Soc. Am., 131, EL302-308 (2012)

First set of results of TREX13 will be documented in a JASA special issue in 2015. 